\title{
A Hybrid Modular Multilevel Converter for Medium-Voltage Variable-Speed Motor Drives
}

\author{
Binbin Li, Student Member, IEEE, Shaoze Zhou, and Dianguo Xu, Senior Member, IEEE, \\ Stephen J. Finney, and Barry W. Williams
}

\begin{abstract}
Modular multilevel converters (MMC) have revolutionized the voltage sourced converter (VSC) based high voltage direct current (HVDC) transmission, but not yet got widespread application in medium-voltage (MV) variable-speed motor drives, because of the large capacitor voltage ripples at low motor speeds. In this paper, a novel hybrid MMC topology is introduced, which significantly reduces the voltage ripple of capacitors, particularly at low motor speeds. Moreover, this topology does not introduce any motor common-mode voltage, as a result there are no insulation and bearing current problems. Additionally the current stress can remain at rated value throughout the whole speed range, thus no device needs to be oversized and converter efficiency can be ensured. Operating principle of this hybrid topology is explained, and control schemes are also developed. Validity and performance of the proposed topology are verified by simulation and experimental results.
\end{abstract}

Index Terms - Capacitor voltage ripple, common-mode voltage, hybrid topology, low motor speed, modular multilevel converter (MMC), motor startup, soft switching, variable-speed drives.

\section{INTRODUCTION}

$\mathrm{M}$ ULTILEVEL inverters for high-power medium-voltage variable-speed motor drives have been widely applied in the industries like compressors, pumps, fans, conveyors, grinding mills, rolling mills, marine propulsion, and rail tractions, to achieve energy savings [1]. Presently, there are mainly four kinds of commercialized multilevel inverter topologies: neutral point clamped (NPC) [2], flying capacitor (FC) [3], cascaded H-bridge (CHB) [4], and the modular multilevel converter (MMC) [5]-[7]. Among them, the MMC, as shown in Fig. 1, is capable of reaching highest voltage and power ratings because: 1) compared to the NPC and FC, MMC features high modularity and scalability, thus voltage level and power rating can be extended easily by increasing the number of sub-modules (SMs), meanwhile reliability can be improved simply by introducing SM redundancies; 2) compared to CHB, the heavy, lossy, costly multi-winding phase-shifted transformer is eliminated, and the MMC also offers a common dc bus configuration.

Nevertheless, since the SM capacitors of MMC are floating,

This work was supported by National Natural Science Foundation of China (51237002) and (51477034), and also by scholarship fund from China Scholarship Council.

B. Li, S. Zhou, and D. Xu are with the School of Electrical Engineering and Automation, Harbin Institute of Technology, Harbin 150001, China (e-mail: libinbinhit@126.com; zhoushaozezsz@foxmail.com; xudiang@hit.edu.cn).

S. Finney and B. Williams are with the Department of Electronics and Electrical Engineering, University of Strathclyde, Glasgow, G1 1XW, U.K. (e-mail: stephen.finney@ strath.ac.uk; barry.williams@strath.ac.uk).

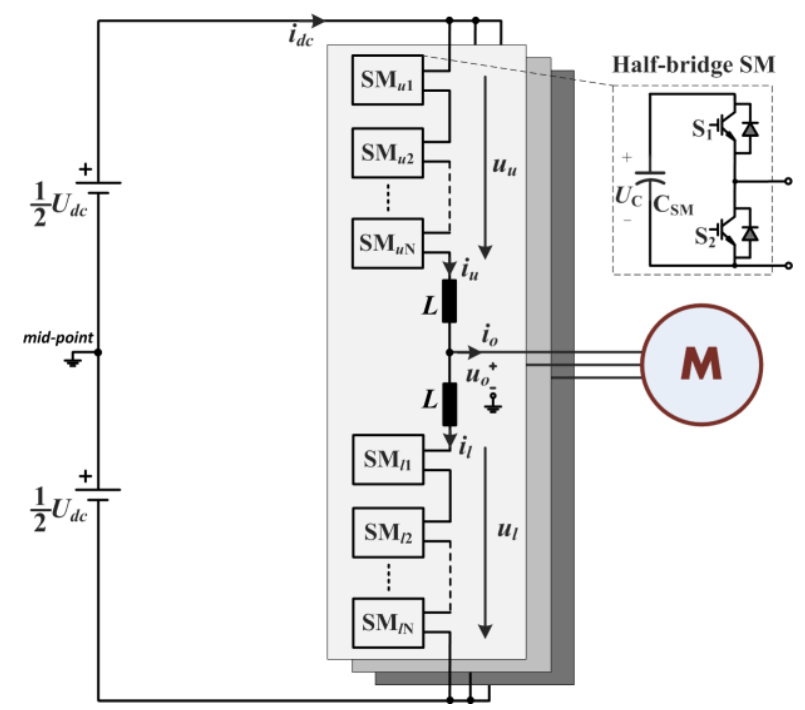

Fig. 1. Circuit configuration of MMC in variable speed drives.

the capacitor voltage will be fluctuated when arm current flows by. This capacitor voltage ripple is theoretically proportional to the output current amplitude and inversely proportional to the output frequency [8]. Hence it is particularly difficult for MMC to drive a constant-torque motor during low speeds. The MMC is more suitable for fan-/blower-like loads, where the load torque is a quadratic function of the motor speed [9]. But usually, a high starting torque is still required to overcome the motor static friction. Therefore, to provide sufficient torque in the low-speed range, measures must be taken to attenuate the SM capacitor voltage ripples. Injection of a high-frequency circulating current into phase-arms of MMC, in coordination with a common-mode voltage of the same frequency imposed at the three-phase ac terminals, is currently the most effective capacitor voltage ripple suppression method [10]-[19]. The injected voltage and current create a high-frequency power exchange between the upper and lower arms, making the SM capacitors get charged and discharged more frequently, so that the voltage ripple can be reduced. The injected common-mode voltage and circulating current waveforms are sinusoidal in [10]-[14]. While the square waveforms [15]-[18], or the inclusion of a third-order current harmonic [19] can also be used to reduce peak value of the injected circulating current to reduce losses. These injection methods, however, have the following limitations and disadvantages:

- High magnitudes of harmful common-mode voltage are superposed upon the motor, resulting in insulation and bearing current problems. Particularly, if the square-wave 


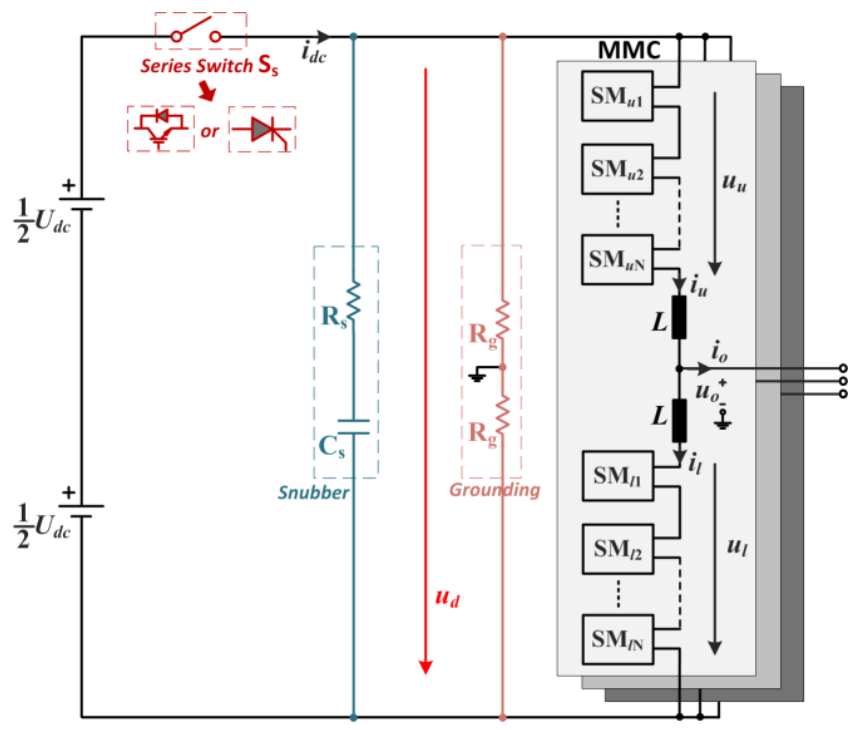

Fig. 2. Circuit configuration of the hybrid modular multilevel converter.

common-mode voltage [15]-[18] is adopted, an additional voltage spike may appear at the motor terminals which is caused by the wave-reflection phenomenon via long cables. These overvoltage and bearing current problems shorten the lifetime of, or even damage, the motor [20].

- High magnitudes of circulating current are injected into the arms of MMC, which significantly increases the current stresses, as well as the power losses, leading to oversized rating values and higher cooling requirements, of the converter.

- Control and coordination of the injected circulating current and common-mode voltage waveforms are quite difficult, and they may also conflict with the basic control loops of MMC (such as the output current control and voltage balancing control [9]), causing instability problems especially during transient processes.

To overcome the above limitations of traditional MMC in variable-speed drives, this paper proposes a new hybrid MMC topology which combines the traditional MMC with a dc link series switch. The main advantage of this topology is the inherently lower capacitor voltage ripple, particularly at low motor speeds. Moreover, no common-mode voltage is imposed at the motor terminals, as a result, there are no insulation and bearing current problems. And the current stress can remain the rated value over the entire speed range, thus no device needs to be oversized and efficiency can be ensured. Therefore, the proposed hybrid MMC is very suitable for motor drives.

The rest of this paper is organized as follows. In Section II the proposed hybrid MMC topology and its operating principle are illustrated. This is followed by the circuit analysis in Section III, which presents why capacitor voltage ripple can be reduced compared to traditional MMC and what are the requirements of devices used in this topology. Further, Section IV discusses the control schemes for this hybrid MMC. Simulation and experimental results are then provided in Section $\mathrm{V}$ to demonstrate validity of the proposed topology and control schemes. Finally, Section VI concludes this paper.

\section{THE HYBRID MMC AND ITS OPERATING PRINCIPLE}

\section{A. The Hybrid MMC Topology}

Circuit configuration of the proposed hybrid MMC is shown in Fig. 2. Between the MMC converter and the dc voltage source, a series switch $S_{s}$, is inserted. With this series switch, MMC converter can be connected to or disconnected from the dc source. Either IGBT or thyristor can be used as this series switch. Besides, a RC snubber circuit [21] is paralleled with the MMC to filter switching voltage harmonics. Two grounding resistors $R_{g}$, are utilized to ground the converter to earth.

With respect to the well-known MMC circuit, each phase of it consists of two arms, the upper and the lower, which are connected through two arm inductors $L$. Each arm is formed by a total of $N$ SMs, and each SM is a half-bridge circuit. The terms $u_{o}$ and $i_{o}$ are the output phase voltage and current, $u_{d}$ is the dc terminal voltage of MMC, $u_{u}, i_{u}$, and $u_{l}, i_{l}$ represent the voltage and current of the upper and the lower arm, respectively. $U_{d c}$ is the dc source voltage and $i_{d c}$ is the dc source current. The SM capacitor voltages are with an average voltage of $U_{C}=U_{d c} / N$. According to Kirchhoff's voltage law, the following voltage relationships exist:

$$
\begin{gathered}
u_{o}=\frac{1}{2}\left(u_{l}-u_{u}-L \frac{d i_{o}}{d t}\right) \\
u_{d}=u_{l}+u_{u}+2 L \frac{d i_{c}}{d t}
\end{gathered}
$$

where $i_{c}$ is defined as the phase circulating current, given by $i_{c}=$ $1 / 2\left(i_{u}+i_{l}\right)$. Moreover, the dc source current $i_{d c}$ can be calculated by summation of the three-phase circulating currents.

The output current $i_{\mathrm{o}}$ of MMC splits equally into the upper and lower arms. Incorporating with the circulating current component $i_{c}$, currents through the upper and lower arms can be expressed as

$$
\left\{\begin{array}{l}
i_{u}=i_{c}+1 / 2 i_{o} \\
i_{l}=i_{c}-1 / 2 i_{o}
\end{array} .\right.
$$

The output ac voltage and current can be written as

$$
\begin{gathered}
u_{o}=U_{O M} \cos (\omega t) \\
i_{o}=I_{O M} \cos (\omega t-\varphi)
\end{gathered}
$$

where $U_{\mathrm{OM}}$ and $I_{\mathrm{OM}}$ are the amplitudes, $\omega$ is the output angular frequency, and $\varphi$ is the phase lag angle.

The output voltage amplitude $U_{\mathrm{OM}}$ can also be expressed by

$$
U_{O M}=1 / 2 m U_{d c}
$$

in which $m$ denotes the modulation index varying from 0 to 1 .

When the converter is used in motor drive applications, a constant $m / \omega$ ratio (i.e., constant Volt/Hertz) should always be ensured. Assuming $m=1$ at the rated motor speed $\omega_{\text {rated }}$, the modulation index at an arbitrary motor speed can be derived as

$$
m=\frac{\omega}{\omega_{\text {rated }}} .
$$

If the series switch $\mathrm{S}_{\mathrm{s}}$ is kept closed, $u_{d}$ equals $U_{d c}$, and the hybrid MMC operates exactly the same as a traditional MMC. The average arm voltages can be approximated by 


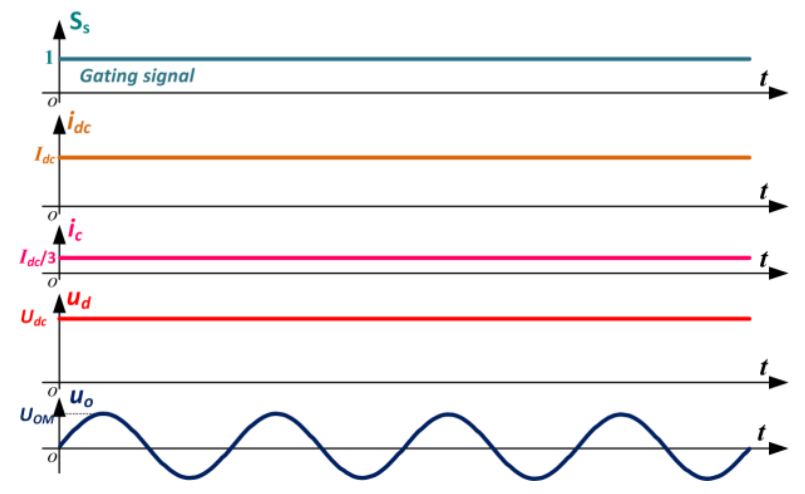

Fig. 3. Sketch map of the operation waveforms of traditional MMC.

$$
\left\{\begin{array}{l}
u_{u}=1 / 2 U_{d c}(1-m \cos (\omega t)) \\
u_{l}=1 / 2 U_{d c}(1+m \cos (\omega t))
\end{array}\right.
$$

and the main waveforms of traditional MMC are presented in Fig. 3. With appropriate even-order harmonics suppression control [22], $i_{c}$ and $i_{d c}$ are both dc currents, with the values of $I_{d c} / 3$ and $I_{d c}$, respectively. According to power balance between dc input and ac output (i.e., $U_{d c} I_{d c}=3 / 2 U_{\mathrm{OM}} I_{\mathrm{OM}} \cos \varphi$ ), $I_{d c}$ can be calculated as

$$
I_{d c}=3 / 4 m I_{O M} \cos \varphi .
$$

This equation shows that, the higher the motor speed $(m)$, or the higher the output torque $\left(I_{\mathrm{OM}}\right)$, the larger the $I_{d c}$. At rated motor speed $(m=1)$ with rated output torque $\left(I_{\mathrm{OM}(\mathrm{rated})}\right), I_{d c}$ reaches its highest value: $I_{d c(\text { rated })}=3 / 4 I_{O M(\text { rated })} \cos \varphi$.

\section{B. Operating Principle of the Hybrid MMC}

Since traditional MMC is only suitable for high motor speeds, during low speeds, the hybrid MMC is controlled as shown in Fig. 4. The series switch $S_{\mathrm{s}}$ turns on and off at the frequency of $f_{h}\left(f_{h}=1 / T_{h}\right)$ with duty cycle of $D$. When $\mathrm{S}_{\mathrm{s}}$ is on, $u_{d}$ equals $U_{d c}$, and the circulating current $i_{c}$ is regulated at $1 / 3 I_{\mathrm{dc}(\text { rated) }}$, thus $i_{d c}$ is equal to $I_{\mathrm{dc}(\text { rated). }}$. When $\mathrm{S}_{\mathrm{s}}$ is off, $u_{d}$ is controlled as $2 U_{\mathrm{OM}}$, while $i_{c}$ and $i_{d c}$ are zero. The currents $i_{d c}$ and $i_{c}$ are expressed as

$$
i_{d c}=\left\{\begin{array}{ll}
I_{d c(\text { rated })} & \text { if }\left(\mathrm{S}_{\mathrm{s}}=1\right) \\
0 & \text { if }\left(\mathrm{S}_{\mathrm{s}}=0\right)
\end{array} \text { and } \quad i_{c}=\frac{1}{3} i_{d c} .\right.
$$

Based on (6), $u_{d}$ can then be represented as

$$
u_{d}= \begin{cases}U_{d c} & \text { if }\left(\mathrm{S}_{\mathrm{s}}=1\right) \\ m U_{d c} & \text { if }\left(\mathrm{S}_{\mathrm{s}}=0\right)\end{cases}
$$

and the average voltage of $u_{d}$ is

$$
\bar{U}_{d}=D U_{d c}+(1-D) m U_{d c} .
$$

The arm voltages of hybrid MMC are

$$
\left\{\begin{array}{l}
u_{u}=1 / 2 u_{d}-1 / 2 m U_{d c} \cos (\omega t)-\Delta u_{d} \\
u_{l}=1 / 2 u_{d}+1 / 2 m U_{d c} \cos (\omega t)-\Delta u_{d}
\end{array} .\right.
$$

That is, comparing (13) with (8), the ac voltage component in arm voltage is not changed. However, the average dc voltage component is reduced, thereby achieving smaller SM capacitor voltage ripple. The detailed voltage ripple analysis will be given in the next section.

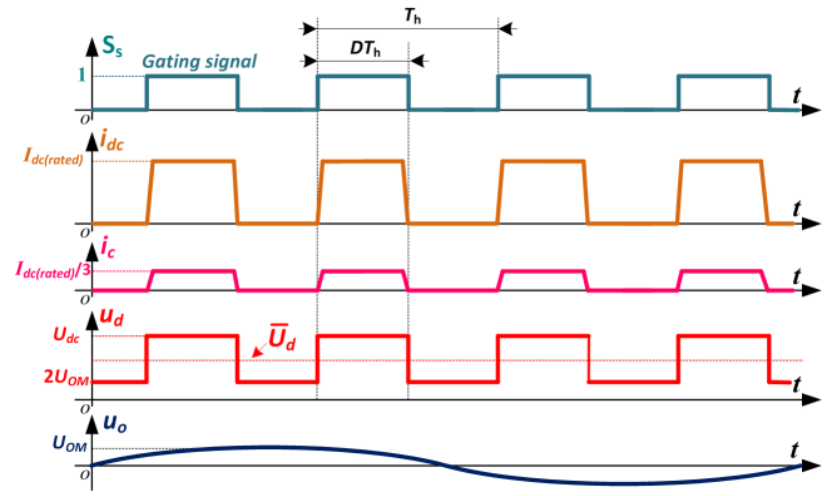

Fig. 4. Sketch map of the operation waveforms of hybrid MMC.

Inserting (13) into (2) yields

$$
L \frac{d i_{c}}{d t}=\Delta u_{d}
$$

in which $\Delta u_{d}$ is used for driving the circulating current $i_{c}$. Due to this current controllability, zero-current switching (ZCS) of the series switch $S_{\mathrm{s}}$ can be realized. Hence a thyristor type device can also be used as this series switch. Considering the arm inductance in motor drives is usually small, in the following analysis the transition time of $i_{\mathrm{c}}$ is omitted and a square $i_{\mathrm{c}}$ waveform is assumed, for simplicity.

The power balance must be ensured in hybrid MMC, which gives $U_{d c} I_{\mathrm{dc}(\text { rated })} D=3 / 2 U_{\mathrm{OM}} I_{\mathrm{OM}} \cos \varphi . D$ is designed equal to 1 at rated output power and rated motor speed, which gives

$$
D=\frac{m I_{O M}}{I_{O M(\text { rated })}} .
$$

Furthermore, if the motor is driving a constant-torque load, where the torque demanded by load is constant throughout the speed range (i.e., $I_{O M}=I_{O M(\text { rated })}$ ), Eq. (15) can be simplified to

$$
D=m \text {. }
$$

\section{CiRCUIT ANALYSIS}

\section{A. Capacitor Voltage Ripple of Traditional MMC}

Based on (3) and (8), the instantaneous power absorbed by each arm of traditional MMC can be calculated as

$$
\left\{\begin{array}{l}
p_{u}=u_{u} i_{u}=\left(1 / 2 U_{d c}-u_{o}\right) \cdot\left(i_{c}+1 / 2 i_{o}\right) \\
p_{l}=u_{l} i_{l}=\left(1 / 2 U_{d c}+u_{o}\right) \cdot\left(i_{c}-1 / 2 i_{o}\right)
\end{array} .\right.
$$

Performing integration of (17) and simplifying, the energy variation of each arm can be derived as (18).

As in motor drive applications, a near constant $m / \omega$ is always guaranteed, while $U_{d c}$ is fixed, and if a constant-torque load is driven, $I_{\mathrm{OM}}$ also remains constant. Then when the motor speed $\omega$ decreases,

- the first term of (18) becomes larger;

- the second term becomes smaller; and

- the third term remains constant.

Hence under low motor speeds, the first energy variation term becomes dominant, whereas the last two terms can be neglected, thus the peak-to-peak energy variation of each arm can be approximated as 


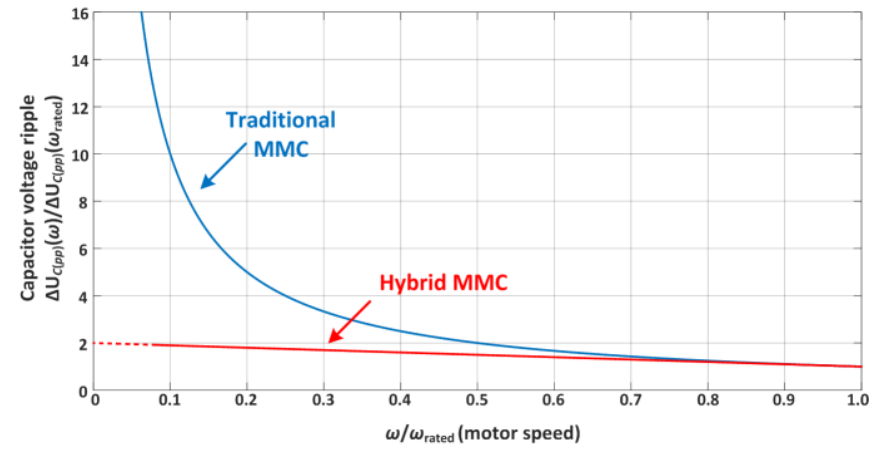

Fig. 5. Normalized capacitor voltage ripple $\Delta U_{C(p p)}(\omega) / \Delta U_{C(p p)}\left(\omega_{\text {rated }}\right)$ of traditional MMC and hybrid MMC in dependence of the motor speed.

$$
\Delta w_{u} \approx \Delta w_{l} \approx \frac{U_{d c} I_{O M}}{2 \omega} .
$$

This energy variation has to be buffered by the SM capacitors in each arm, that is

$$
\frac{U_{d c} I_{O M}}{2 \omega}=N\left(1 / 2 C U_{C(\max )}^{2}-1 / 2 C U_{C(\min )}^{2}\right)=N C U_{C} \Delta U_{C(p p)}
$$

where $\Delta U_{C(p p)}$ is the peak-to-peak SM capacitor ripple voltage.

Since $N U_{C}=U_{d c}$, Eq. (20) can then be simplified to

$$
\Delta U_{C(p p)}=\frac{I_{O M}}{2 \omega C} \text {. }
$$

Clearly, it is shown that capacitor voltage ripple of traditional MMC is proportional to the output torque and inversely proportional to the motor speed. As such, with high torque requirements at low speeds, the ripple voltage would be unacceptably large.

\section{B. Capacitor Voltage Ripple of Hybrid MMC}

With respect to the proposed hybrid MMC, its unique feature is that the capacitor voltage ripple can be significantly reduced. The basic idea comes from (19), as $I_{\mathrm{OM}}$ is determined by load torque, the only way to avoid large energy variation is to reduce the dc component of the arm voltages, $1 / 2 U_{d c}$. This is achieved in hybrid MMC by disconnecting with the dc source at regular intervals and generating the arm voltages as given in (13). Consequently, the average dc arm voltage component can be reduced to $1 / 2 \bar{U}_{d}$.

Similar to the previous analysis, energy variation of each arm of hybrid MMC can be derived as

$$
\Delta w_{u} \approx \Delta w_{l} \approx \frac{(D+(1-D) m) U_{d c} I_{O M}}{2 \omega} .
$$

Thus the capacitor voltage ripple is

$$
\begin{aligned}
& \Delta U_{C(p p)}=\frac{(D+(1-D) m) I_{O M}}{2 \omega C} .
\end{aligned}
$$

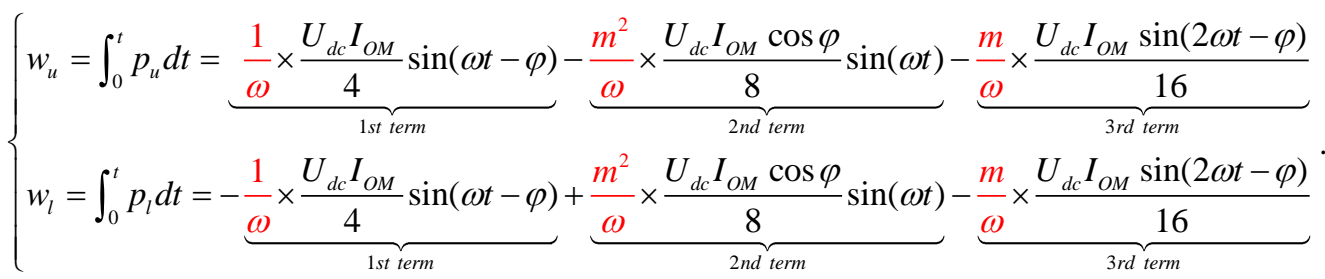

Inserting (7) and (15) into (23) gives

$$
\Delta U_{C(p p)}=\frac{\left[I_{O M(\text { rated })}+\left(1-\frac{\omega}{\omega_{\text {rated }}}\right) I_{O M}\right] I_{O M}}{2 \omega_{\text {rated }} I_{O M \text { (rated })} C} .
$$

Particularly, for the case of constant-torque load with $I_{\mathrm{OM}} \equiv I_{\mathrm{OM}(\text { rated) }}$, (24) can be further simplified to

$$
\Delta U_{C(p p)}=\left(2-\frac{\omega}{\omega_{\text {rated }}}\right) \frac{I_{O M(\text { rated })}}{2 \omega_{\text {rated }} C} .
$$

The detailed comparison of equations (21) and (25) at different motor speed is illustrated in Fig. 5. It can be seen that, in contrast to traditional MMC, capacitor voltage ripple of hybrid MMC at low speeds is significantly reduced. Although the voltage ripple of hybrid MMC also increases when the motor speed decreases, its maximum voltage ripple at very low speeds is only up to twice of the value at rated speed.

As a consequence, by allowing a larger capacitor voltage ripple when designing the SM capacitor (i.e., making allowance for a higher ripple voltage margin when determining the capacitor's maximum operating peak voltage), the hybrid MMC can produce rated motor torque throughout the whole speed range. On the other hand, if the motor load is not the constant-torque type, for example, the most common loads in industries are centrifugal pumps and fans which are quadratic-torque type, capacitor voltage ripple of hybrid MMC at different motor speeds can be solved using (24).

\section{The Possibility of Operating at Zero Motor Speed}

It is worth noting that, from (25), it shows even the motor speed is zero (i.e., $\omega=0$ ), capacitor voltage ripple is still limited. But practically, at speeds very close to zero (below $2 \mathrm{~Hz}$ ), the Volt/Hertz relationship of motor would not be satisfied, and the duty cycle $D$ will be so small that it approaches the time limit for current control in (14). Therefore, the capacitor voltage ripple in this case will be much larger than the above theoretical analysis. And it is not possible for the hybrid MMC to drive a motor stably operating at zero speed with a high torque request.

However, most medium-voltage motors are not required to operate at zero speed. Due to the acceleration of motor, motor speed will quickly surpass the close-to-zero range, (and with an asynchronous machine, the slip frequency aspect relieves this problem) so the hybrid MMC can be effective to start up a motor from standstill.

\section{Component Requirements of Hybrid MMC}

1) The series switch $S_{s}$ :

The current stress of $S_{s}$ is the amplitude of dc source current $i_{d c}$, which is, according to (10), always $I_{d c(\text { rated })}$. Moreover, when 


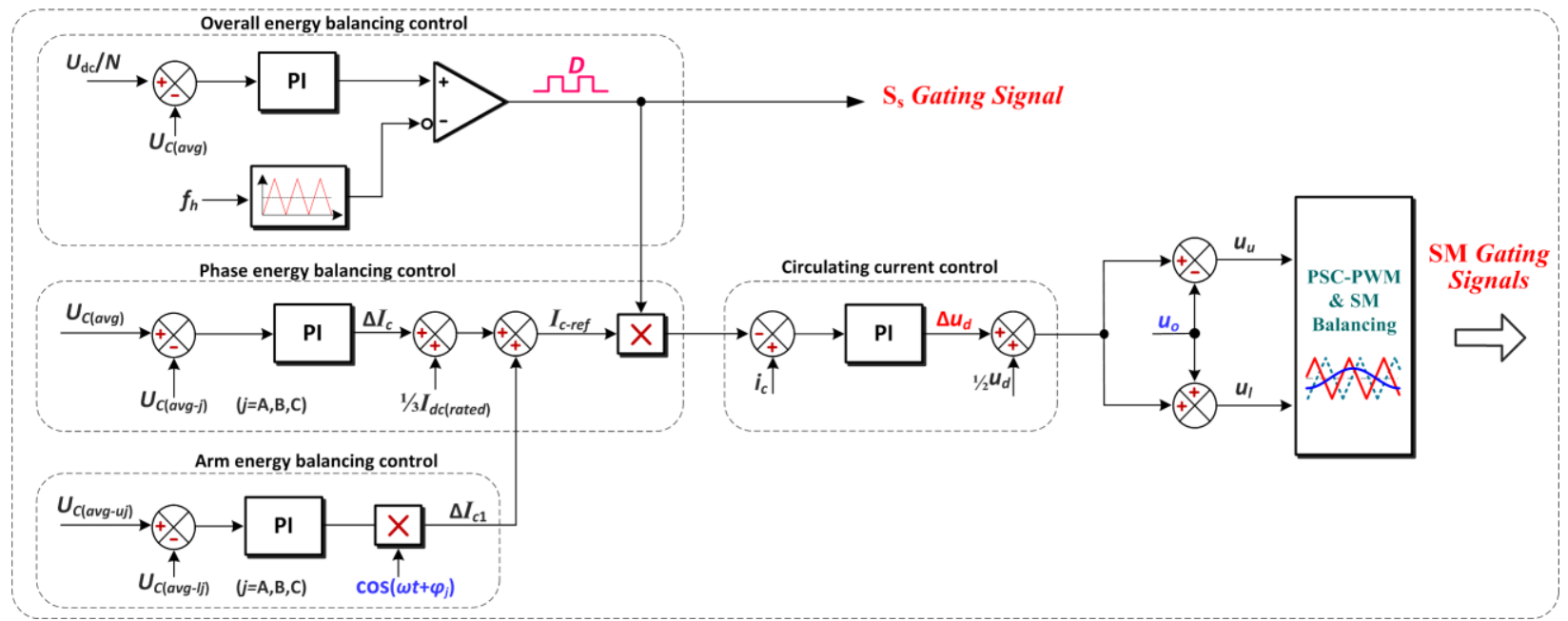

Fig. 6. Control block diagrams for the hybrid MMC.

$\mathrm{S}_{\mathrm{s}}$ commutates, $i_{d c}$ is able to be maintained at zero by the MMC circuit, which means zero-current switching (ZCS) is realized and the switching loss of $\mathrm{S}_{\mathrm{s}}$ is small. Again as $i_{d c}$ can be controlled to zero before $S_{\mathrm{s}}$ turns off, not only the IGBT, but also the more robust thyristor can be employed.

The voltage that to be blocked by $\mathrm{S}_{\mathrm{s}}$ is the voltage difference between dc source and MMC circuit, which is, according to (11), (1-m) $U_{d c}$. As $m$ would be very small when the speed is low, the voltage rating of $\mathrm{S}_{\mathrm{s}}$ has to be higher than $U_{d c}$. In medium-voltage applications, this switch implementation will require series connection of devices (IGBTs or IGCT thyristors). In this case a main challenge has been to ensure voltage sharing between the devices. Methods like adopting paralleled resistors for static voltage balancing, using snubbers for dynamic voltage balancing, and employing gate control for active voltage balancing, should be considered [23]-[25]. However, due to the soft-switching capability in hybrid MMC (zero current and around zero voltage), implementation of the series connection will be easier.

Besides, if the motor mainly operates at high speeds but only needs to provide a high starting torque during startup, a mechanical switch can be paralleled to bypass $S_{\mathrm{s}}$ when motor reaches its operational speed, then the hybrid MMC operates exactly as a traditional MMC. In this way, conduction loss of $\mathrm{S}_{\mathrm{s}}$ can be further eliminated.

2) The SM IGBTs:

Different from the existing circulating current injection methods, the arm current amplitude of hybrid MMC can remain the rated value $\left(1 / 2 I_{O M(\text { rated })}+1 / 3 I_{d c \text { (rated })}\right)$ within the whole speed range, thus the SM IGBTs do not need to be oversized thus cost can be saved. In addition, the rms values of the arm currents of traditional MMC and hybrid MMC can be respectively expressed as

$$
\left\{\begin{array}{l}
I_{\text {rms-tra-MMC }}=\sqrt{D^{2}\left(\frac{\left.I_{d c(\text { rated })}\right)^{2}+1 / 2 I_{O M(\text { rated })}{ }^{2}}{3}\right)^{2}+1 / 2 I_{O M(\text { rated })}^{2}} \\
I_{\text {rms-hybrid-MMC }}=\sqrt{D\left(\frac{I_{d c(\text { rated })}}{3}\right)^{2}} .
\end{array}\right.
$$

Since $D \leq 1$, rms value of hybrid MMC is larger than traditional MMC during low speeds (when $D<1$ ), which means the SM power loss in hybrid MMC is slightly higher. But the maximum rms values of (26) (when $D=1$, i.e., at rated motor speed and rated power) are equal. As a result, cooling requirements of SMs in hybrid MMC do not need to be increased.

\section{3) Motor stress:}

Another desirable feature of hybrid MMC is the avoidance of causing harm to the motor. Because the output voltages imposed at motor terminals are sinusoidal, and do not introduce any harmful common-mode voltage. Therefore, the motor has no insulation and bearing current problems. This eliminated common-mode voltage is of crucial importance in the variable-speed drive applications, to ensure motor's lifespan.

4) Filters and snubber circuit:

Since switching of the series switch $\mathrm{S}_{\mathrm{s}}$ causes discontinuous dc current, additional filtering should be considered at the dc input, to limit the current harmonic with frequency of $f_{h}$.

Besides, it should be noted if $\mathrm{S}_{\mathrm{s}}$ adopts IGBT but ZCS switching is not realized (i.e., the IGBT turns off before the current drops to zero), then a voltage spike of opposite polarity may occur at $u_{d}$ due to electromagnetic induction of the arm inductances. Although the RC snubber circuit can suppress this spike to some extent, when the arm current at the instant of turning-off is large, this voltage spike could still be high. In this case, a reverse paralleled diode or a metal-oxide varistor (MOV) should be added to the RC snubber circuit to avoid overvoltage.

\section{CONTROL SCHEMES}

This section develops the suitable control schemes for the hybrid MMC. As summarized in Fig. 6, the complete control system consists of the following five main controls: overall energy balancing control, phase energy balancing control, arm energy balancing control, circulating current control, and pulse-width modulation (PWM) associated with SM capacitor voltage balancing.

In order to achieve stable operation of the hybrid MMC, power balance between dc input and ac output must be 


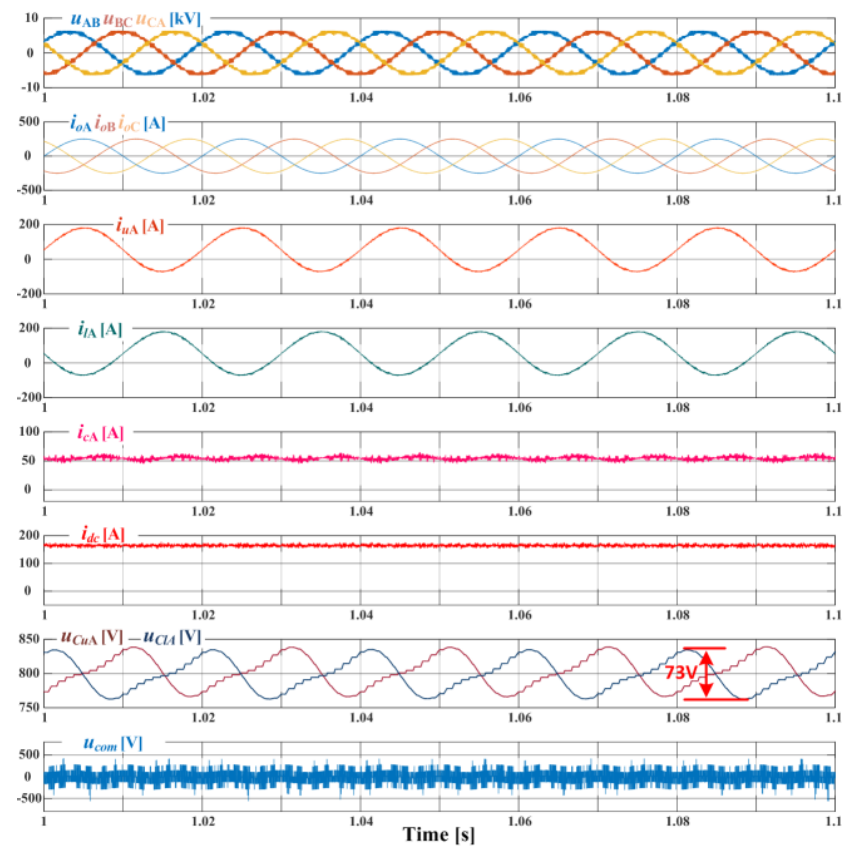

Fig. 7. Simulation waveforms of traditional MMC at $50 \mathrm{~Hz}$.

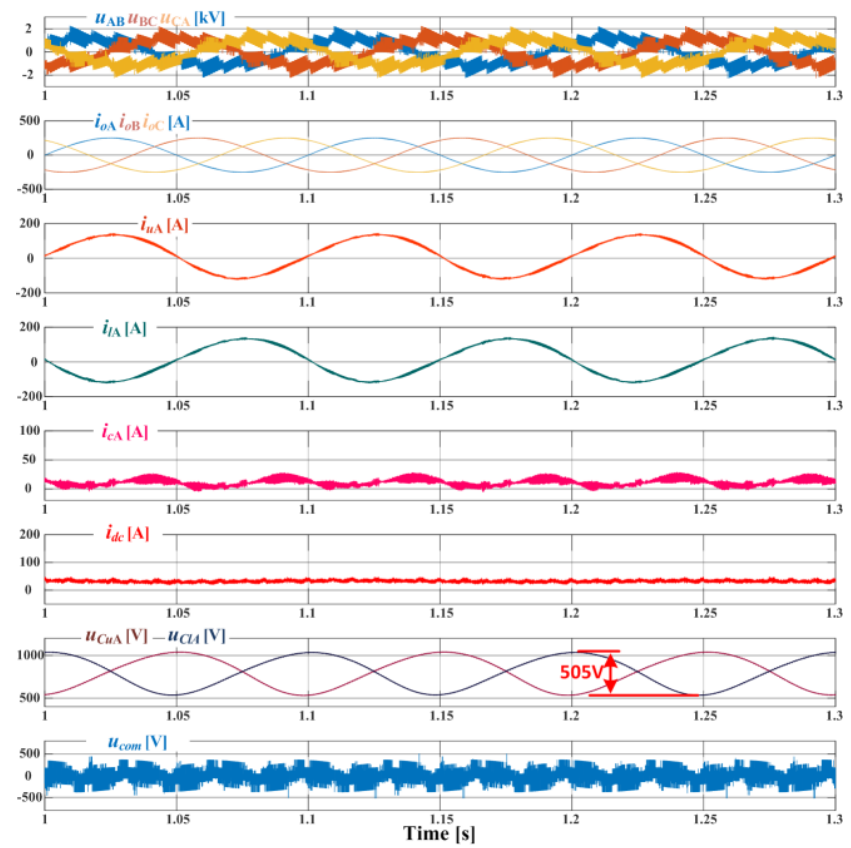

Fig. 8. Simulation waveforms of traditional MMC at $10 \mathrm{~Hz}$.

maintained. The difference between the input and output powers would influence the stored energy in the capacitors, so an overall energy balancing control is used to keep the measured average voltage of all the SM capacitors $U_{C(\text { avg })}$ equal to the reference value $U_{d c} / N$. This is accomplished by adjusting the duty cycle $D$.

Moreover, a phase energy balancing control is adopted to ensure the total energy are evenly distributed among the three
TABLE I

SIMULATION PARAMETERS

\begin{tabular}{c|c}
\hline \hline \multicolumn{2}{c}{ Hybrid MMC parameters } \\
\hline Number of SMs per arm & $N=10$ \\
\hline DC-source voltage & $U_{\mathrm{dc}}=8000 \mathrm{~V}$ \\
\hline Nominal SM capacitor voltage & $U_{C}=800 \mathrm{~V}$ \\
\hline SM capacitance & $C_{\mathrm{SM}}=4000 \mu \mathrm{F}$ \\
\hline Arm inductance & $L=1 \mathrm{mH}$ \\
\hline Rated output frequency & $f_{o(\text { rated })}=50 \mathrm{~Hz}$ \\
\hline Rated phase current magnitude & $I_{O M(\text { rated })}=250 \mathrm{~A}$ \\
\hline Rated dc current & $I_{d c \text { (rated })}=180 \mathrm{~A}$ \\
\hline PSC-PWM frequency & $f_{\mathrm{c}}=1 \mathrm{kHz}$ \\
\hline Series switch & IGBT (two in series $)$ \\
\hline S $_{\mathrm{s}}$ switching frequency & $f_{h}=10 \times f_{\mathrm{o}}$ \\
\hline Snubber resistance & $200 \Omega$ \\
\hline Snubber capacitance & $1 \mu \mathrm{F}$ \\
\hline \multicolumn{2}{c}{ RL load parameters } \\
\hline Load resistance & $R_{\text {load }}=14 \times f_{\mathrm{o}} / 50 \Omega$ \\
\hline Load inductance & $L_{\text {load }}=2 \mathrm{mH}$ \\
\hline \multicolumn{2}{c}{ PMSM parameters } \\
\hline Rated active power & $P=1.3 \mathrm{MW}$ \\
\hline Number of pole pairs & $p p=10$ \\
\hline Rated rotor speed & $n_{\text {rated }}=300 \mathrm{rpm}$ \\
\hline Rated rms line-to-line voltage & $U_{\text {rated }}=4.16 \mathrm{kV}$ \\
\hline Mechanical load torque & $T=40000 \mathrm{~N}$ \\
\hline \hline
\end{tabular}

phases. The measured average capacitor voltage of each phase $U_{C(\text { avg-j) }}$ ( $j$ represents phase A, B, C, respectively) is controlled equal to $U_{C(\text { avg })}$, by producing a dc current adjusting component $\Delta I_{c}$.

Further, an arm energy balancing control is utilized to maintain the energy in each phase equally distributed between upper and lower arms [26]. This is realized by adjusting a fundamental-frequency current component $\Delta I_{c 1}$. Subsequently these current adjusting components are added with $1 / 3 I_{d c(\text { rated })}$, which generates $I_{c \text {-ref }}=1 / 3 I_{d c(\text { rated })}+\Delta I_{c}+\Delta I_{c 1} . \quad I_{\text {c-ref }}$ is then multiplied by duty cycle $D$, obtaining the circulating current reference. The objective of using circulating current control is forcing the actual circulating current to follow $I_{\mathrm{c} \text {-ref, }}$, where the controlled variable is $\Delta u_{d}$. According to (13), references for the arm voltages $u_{u}$ and $u_{l}$ can then be obtained.

Finally, references $u_{u}$ and $u_{l}$ are sent to the PWM generator to synthesize a stepped switched voltage waveform. There are many kinds of modulation techniques for MMCs [7]. But due to the limited number of SMs adopted in motor drive applications, the phase-shifted carrier PWM (PSC-PWM) [27], with carriers evenly distributed, is adopted to reduce output voltage harmonics and improve the equivalent switching frequency. Besides, a voltage balancing scheme based on adjustment of the reference signals [9] is adopted to balance the SM capacitor voltages within each arm.

\section{SiMULATION AND EXPERIMENTAL RESUlTS}

A. Simulation Results with RL Loads 


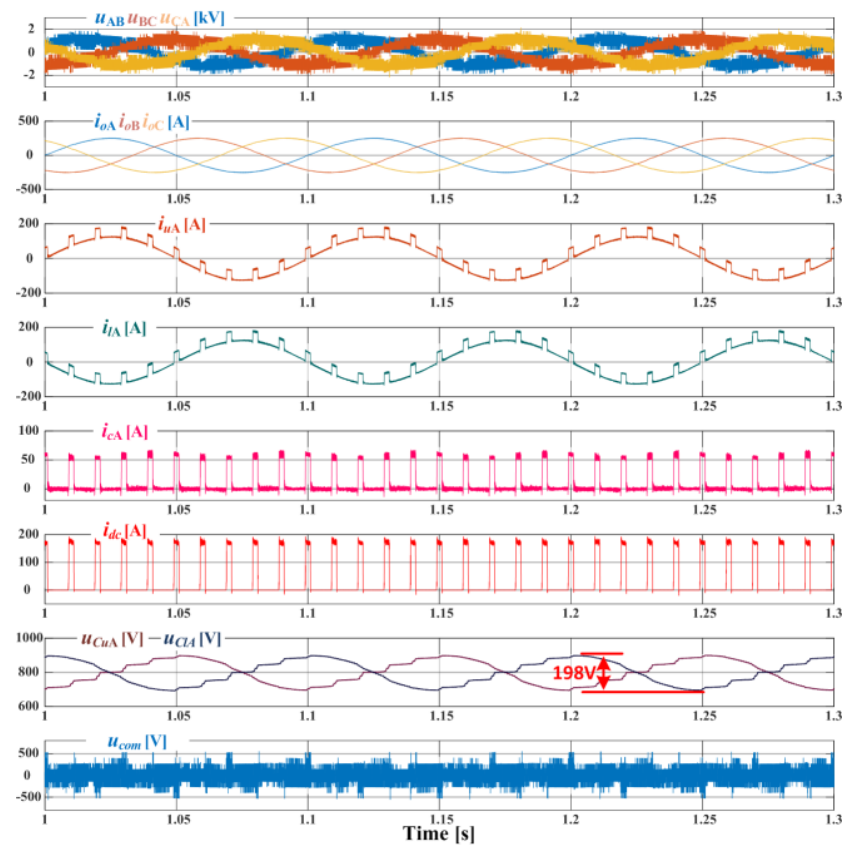

Fig. 9. Simulation waveforms of proposed hybrid MMC at $10 \mathrm{~Hz}$.

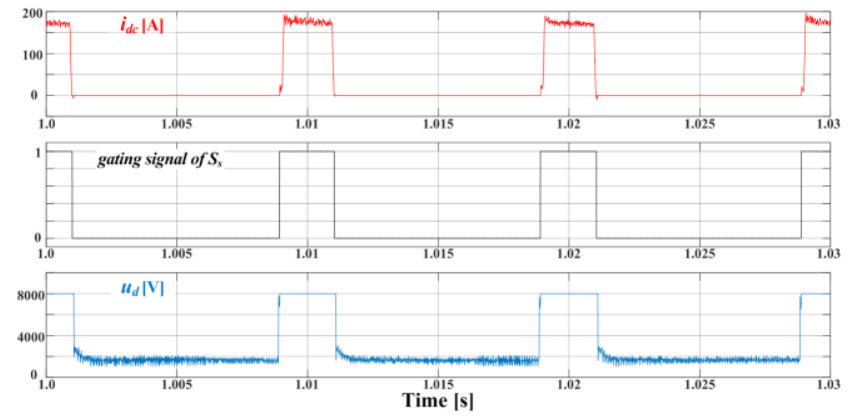

Fig. 10. Zoomed waveforms of series switch of hybrid MMC at $10 \mathrm{~Hz}$.

In order to verify effectiveness of the proposed hybrid MMC topology and control schemes, a 1.3 MW hybrid MMC with two IGBT series switch was simulated, using the MATLAB /Simulink software. The MMC was designed with ten SMs per arm (total of $60 \mathrm{SMs}$ ), and the nominal capacitor voltage was $800 \mathrm{~V}$. Parameters of the simulated circuit and operating conditions are listed in Table I. To start with, the RL load is used to test the steady-state behaviours. The load resistance was varied linearly with the output frequency $\left(R_{\text {load }}=14 \times f_{\mathrm{o}} / 50[\Omega]\right)$ to emulate a motor constant torque characterisitc (with $I_{\mathrm{OM}}=250 \mathrm{~A}$ ).

Figs. 7 and 8 show the operation waveforms of traditional MMC (by having $\mathrm{S}_{\mathrm{s}}$ of hybrid MMC constantly ON), at $50 \mathrm{~Hz}$ and $10 \mathrm{~Hz}$, respectively. In Fig. 7, the dc current $i_{\mathrm{dc}}$ was at its rated value $180 \mathrm{~A}$, and the arm current $\left(i_{u \mathrm{~A}}\right.$ and $\left.i_{\mathrm{IA}}\right)$ amplitudes were also about $180 \mathrm{~A}$ which approximately equals $\left(1 / 2 I_{O M(\text { rated })}+1 / 3 I_{d c(\text { rated })}\right)$. The capacitor voltages $u_{C u A}$ and $\mathrm{u}_{C l A}$ were balanced at $800 \mathrm{~V}$ with a peak-to-peak ripple of $73 \mathrm{~V}(9 \%)$. In Fig. 8, with decrease of the output frequency, ripple of the capacitor voltage was significantly increased, to $505 \mathrm{~V}(63 \%)$. If the output frequency is decreased further, this voltage ripple would be extremely large causing overvoltage problems and the converter can no longer operate.

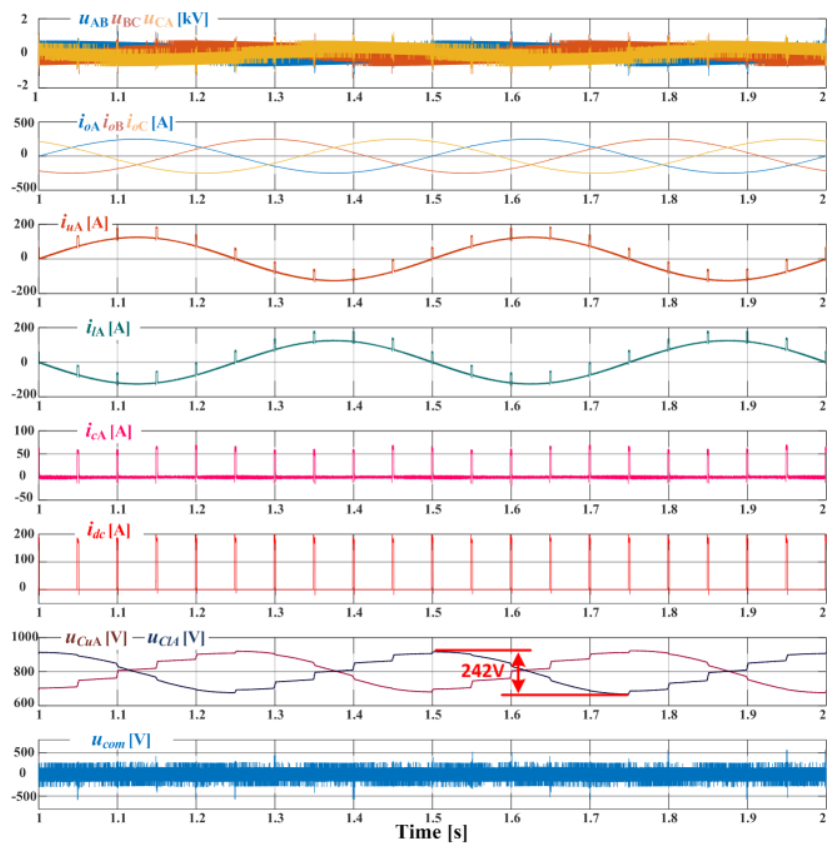

Fig. 11. Simulation waveforms of proposed hybrid MMC at $2 \mathrm{~Hz}$.

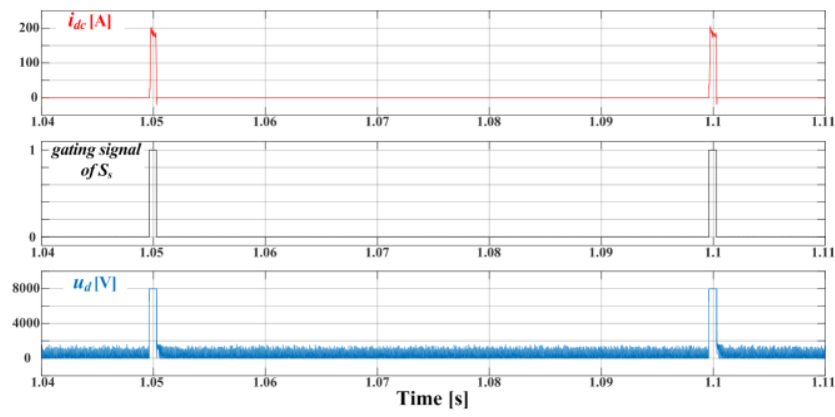

Fig. 12. Zoomed waveforms of series switch of hybrid MMC at $2 \mathrm{~Hz}$.

Noted that the capacitor voltage ripple at $10 \mathrm{~Hz}$ was not five times the value at $50 \mathrm{~Hz}$. This is because the last two terms in Eq. (18), which would couteract some of the stored energy of first term, are not negligible at $50 \mathrm{~Hz}$, resulting in practically smaller capacitor voltage ripple.

Fig. 9 shows the operation waveforms of proposed hybrid MMC at $10 \mathrm{~Hz}$. It is shown that the ac currents were high-quality sinusoidal waveforms, and the SM capacitor voltages were well balanced with an average value of $800 \mathrm{~V}$. Importantly, compared to Fig. 8, the capacitor voltage ripple has been reduced, to $198 \mathrm{~V}(25 \%)$ : confirming the proposed hyrbid MMC has lower capacitor voltage ripple. In Fig. 10, the dc current $i_{d c}$, gating signal of $\mathrm{S}_{\mathrm{s}}$, and the de terminal voltage $u_{d}$ were presented. It can be observed that soft switching of $S_{\mathrm{s}}$ was achieved, in which $i_{d c}$ was maintained small when the gate signal was applied, whereas $i_{d c}$ was brought to zero before the gate signal removal.

Figs. 11 and 12 further present the operation waveforms of hybrid MMC at $2 \mathrm{~Hz}$. Under this very low frequency, the hybrid MMC can still operate with rated output current and the capacitor voltage ripple was only $242 \mathrm{~V}(30 \%)$.

Apart from the significantly reduced capacitor votlage ripple, Figs. 9 and 11 also demenstrated other advantages of hybrid 


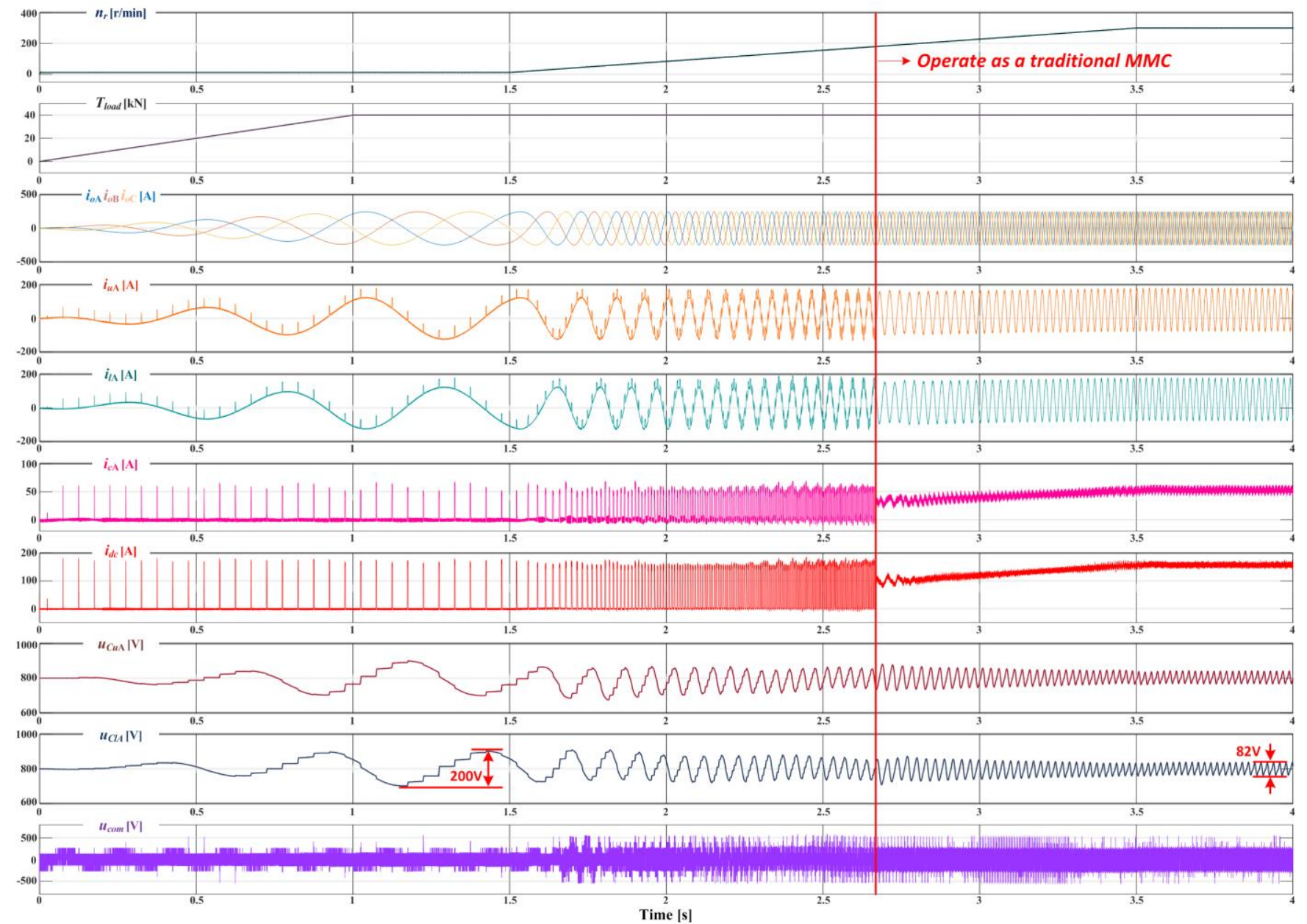

Fig. 13. Simulation waveforms of a PMSM motor driven by hybrid MMC running up from standstill to rated speed, with constant load torque.

MMC: 1) the arm currents and dc current basically remained at their rated values (below 200A), no overcurrent occured thus converter losses were reduced, and current overrating of the converter can be avoided; 2) the common-mode voltage $u_{\text {com }}$ always stayed within $\pm 500 \mathrm{~V}$ and only contained harmonics at switching frequency. No high magnitude of common-mode voltage was intentionally injected, thus no harm was caused to the loads.

\section{B. Simulation Results with PMSM}

To test the dynamic performance of hybrid MMC and the proposed control schemes, a 20 -pole $4.16 \mathrm{kV} / 1.3 \mathrm{MW}$ PMSM with a constant load torque, was simulated, with the motor parameters in Table I. The motor was controlled by an outer speed control loop and an inner vector control loop (with $i_{\mathrm{d}}=0$ ). Fig. 13 shows the complete waveforms of motor run-up from standstill to its rated speed (300r/min). During low speed region $[0,180 \mathrm{r} / \mathrm{min}]$, the converter operated as the hybrid MMC to limit capacitor voltage ripples. After the motor speed exceeded 180r/min, the series switch $\mathrm{S}_{\mathrm{s}}$ was constantly $\mathrm{ON}$ and the hybrid converter turned into traditional MMC. It is clear from Fig. 13 that the dynamic waveforms were smooth with no overshoot, neither in the currents nor the capacitor voltages, and the maximum capacitor voltage ripple was limited as $200 \mathrm{~V}$ (25\%). Moreover, throughout the whole speed range, neither overcurrent problem nor severe common-mode voltage problems have been introduced.

\section{Experimental Results with RL Loads}

A scaled-down hybrid MMC prototype, with three SMs per arm (total of $18 \mathrm{SMs}$ ), has been built in the laboratory to practically verify the effectiveness of the topology. Picture of the prototype is shown in Fig. 14, and its parameters and operating conditions are listed in Table II. Only RL loads were tested in the experiments, in which the load resistance was varied linearly with the output frequency $\left(R_{\text {load }}=40 \times f_{\mathrm{o}} / 50[\Omega]\right)$ so as to emulate the constant torque characterisitc (with $I_{\mathrm{OM}}=5 \mathrm{~A}$ ) of motor. To focus on the capacitor voltage ripple, soft switching of $\mathrm{S}_{\mathrm{s}}$ was not considered and a diode was added in reverse parallel with the RC snubber circuit to avoid overvoltage. The common-mode voltage was observed by measuring voltage at the star-neutral point of the three-phase loads.

Figs. 15 and 16 show the experimental results of traditional MMC (by maintaining $\mathrm{S}_{\mathrm{s}}$ constantly $\mathrm{ON}$ ), at $50 \mathrm{~Hz}$ and $5 \mathrm{~Hz}$, respectively. In Fig. 15, the capacitor voltage ripple was only $3.5 \mathrm{~V}(2.3 \%)$, whereas in Fig. 16 the voltage ripple was significantly increased, to $49.5 \mathrm{~V}(33 \%)$. If the output frequency was further decreased, this voltage ripple would be too large for traditional MMC to operate properly. 


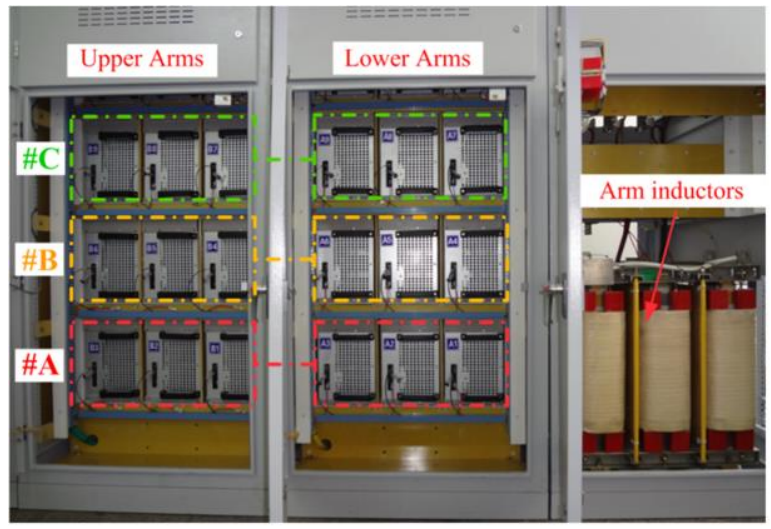

(a) MMC circuits

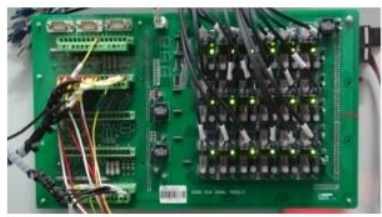

(b) control board

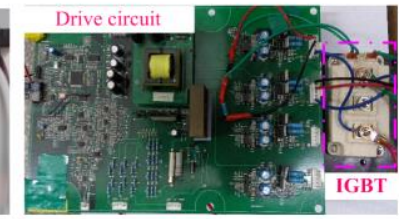

(c) series switch
Fig. 14. Picture of the experimental prototype.

TABLE II

EXPERIMENT PARAMETERS

\begin{tabular}{c|c}
\hline \hline \multicolumn{2}{c}{ Hybrid MMC parameters } \\
\hline Number of SMs per arm & $N=3$ \\
\hline DC-source voltage & $U_{\mathrm{dc}}=450 \mathrm{~V}$ \\
\hline Nominal SM capacitor voltage & $U_{C}=150 \mathrm{~V}$ \\
\hline SM capacitance & $C_{\mathrm{SM}}=1800 \mu \mathrm{F}$ \\
\hline Arm inductance & $L=2 \mathrm{mH}$ \\
\hline Rated output frequency & $f_{o(\text { rated })}=50 \mathrm{~Hz}$ \\
\hline Rated phase current magnitude & $I_{O M(\text { rated })}=5 \mathrm{~A}$ \\
\hline Rated dc current & $I_{d c(\text { rated })}=5 \mathrm{~A}$ \\
\hline PSC-PWM frequency & $f_{\mathrm{c}}=3 \mathrm{kHz}$ \\
\hline Series switch & IGBT(FF300R $12 \mathrm{KE} 3)$ \\
\hline$S_{\mathrm{s}}$ switching frequency & $f_{h}=10 \times f_{\mathrm{o}}$ \\
\hline Snubber resistance & $37 \Omega$ \\
\hline Snubber capacitance & $10 \mu \mathrm{F}$ \\
\hline RL load parameters \\
\hline Load resistance & $R_{\text {load }}=40 \times f_{\mathrm{o}} / 50 \Omega$ \\
\hline Load inductance & $L_{\text {load }}=1.5 \mathrm{mH}$ \\
\hline
\end{tabular}

Fig. 17 shows the experiemental results of hybrid MMC at $5 \mathrm{~Hz}$. In contrast to traditional MMC, capacitor voltage ripple was reduced to $18.5 \mathrm{~V}(12 \%)$. Fig. 18 further presents the experimental waveforms of hybrid MMC at $2 \mathrm{~Hz}$. Under this very low frequency, the hybrid MMC can still operate with rated output current and the capacitor voltage ripple was $26.5 \mathrm{~V}$ $(18 \%)$. These results again proved the hyrbid MMC inherently has lower capacitor voltage ripple. But it should be noted that, the voltage ripple in experiments was much larger than the theroretical value. Because voltage rating of the experimental prototype was too small compared to an actual medium-voltage converter, thus the voltage needed for circulating current control and the voltage drops on SM IGBTs and parasatic resistances were not negligible. As a result, the dc voltage component in (12) has to be higher to make allowance for these voltage drops, which leads to larger capacitor voltage ripple,
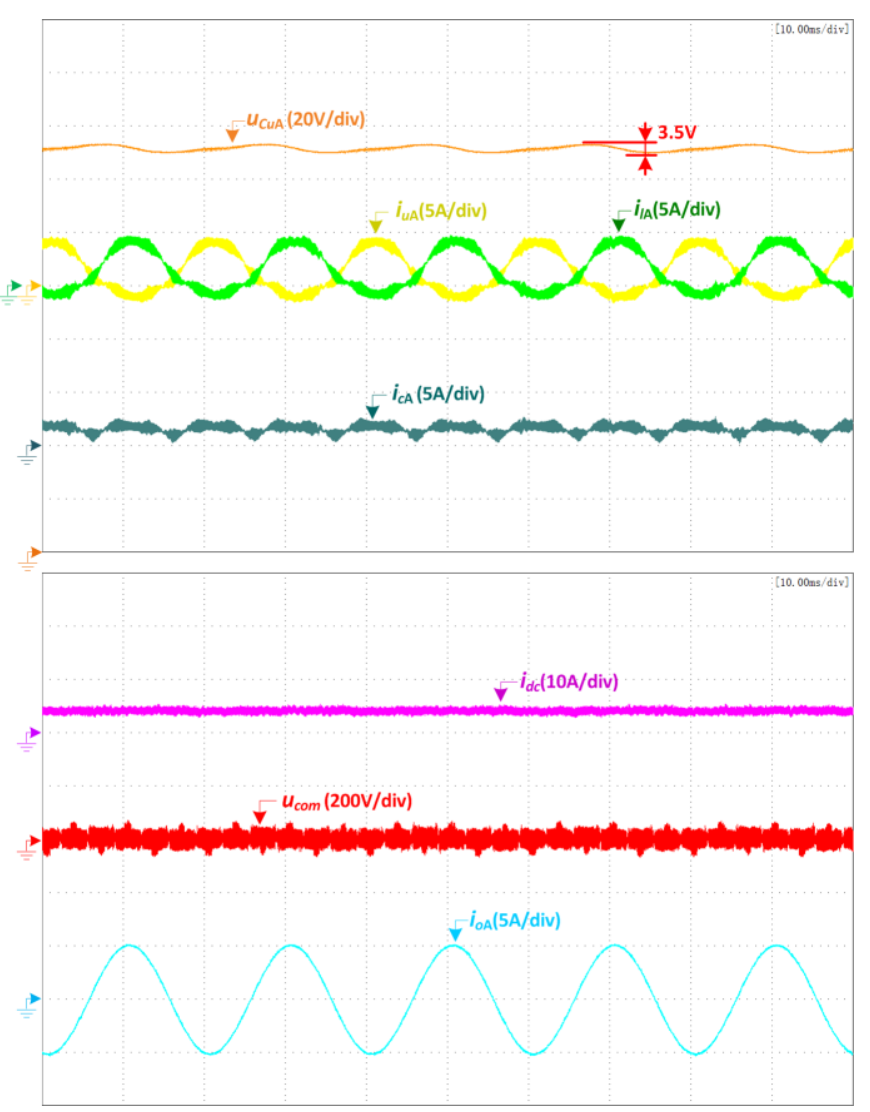

Fig. 15. Experimental waveforms of traditional MMC at $50 \mathrm{~Hz}$.

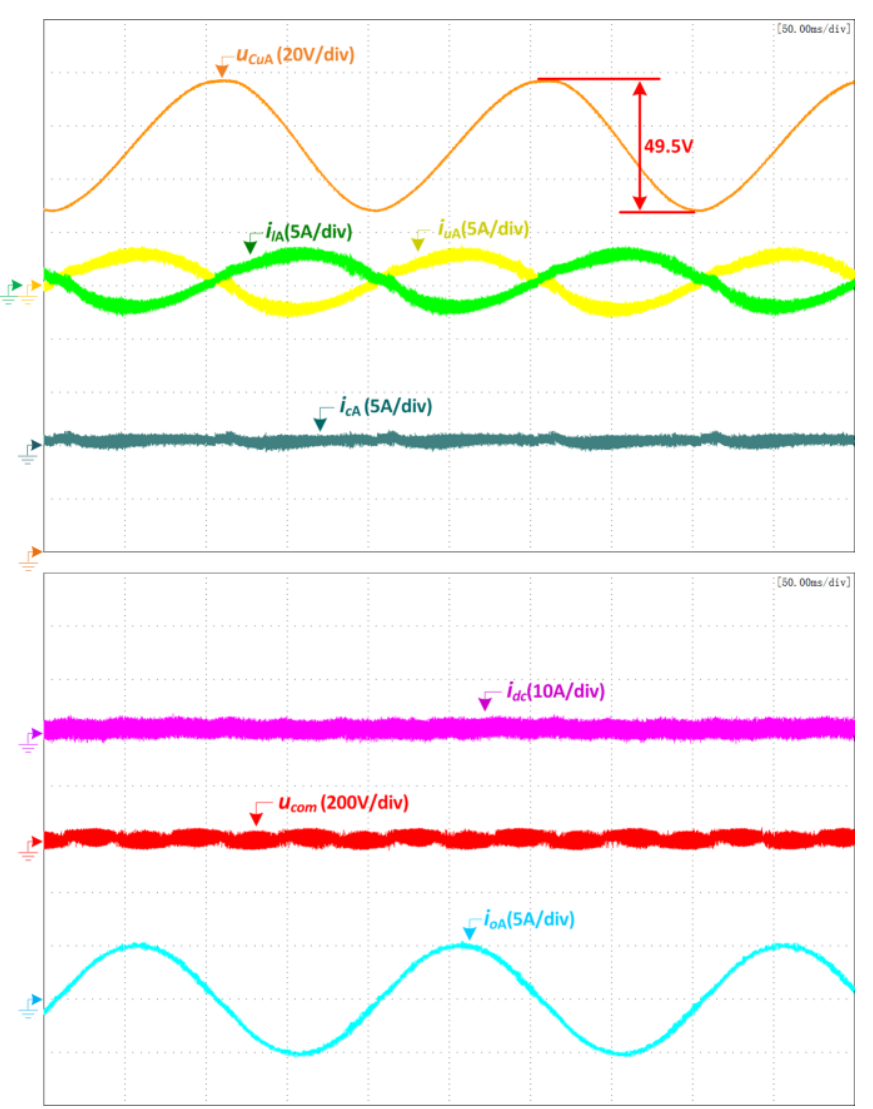

Fig. 16. Experimental waveforms of traditional MMC at $5 \mathrm{~Hz}$.

particularly under low frequencies. 


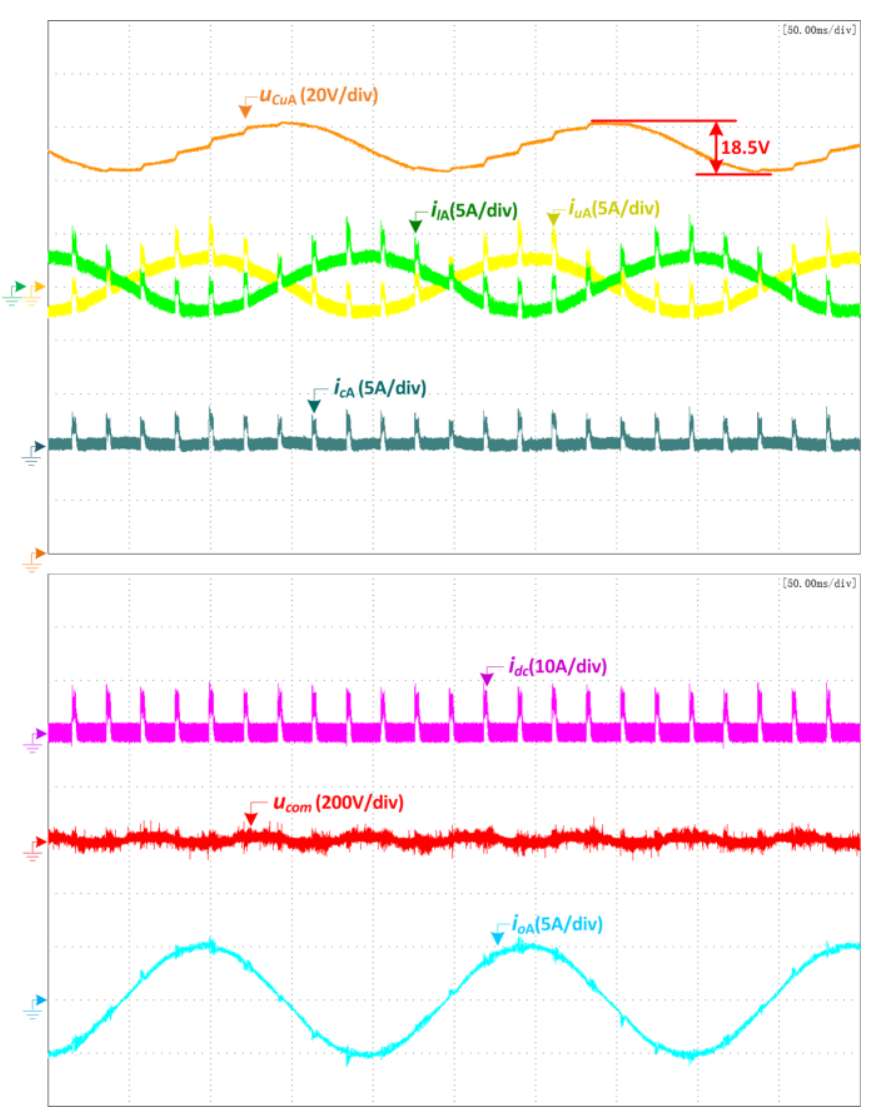

Fig. 17. Experimental waveforms of hybrid MMC at $5 \mathrm{~Hz}$.

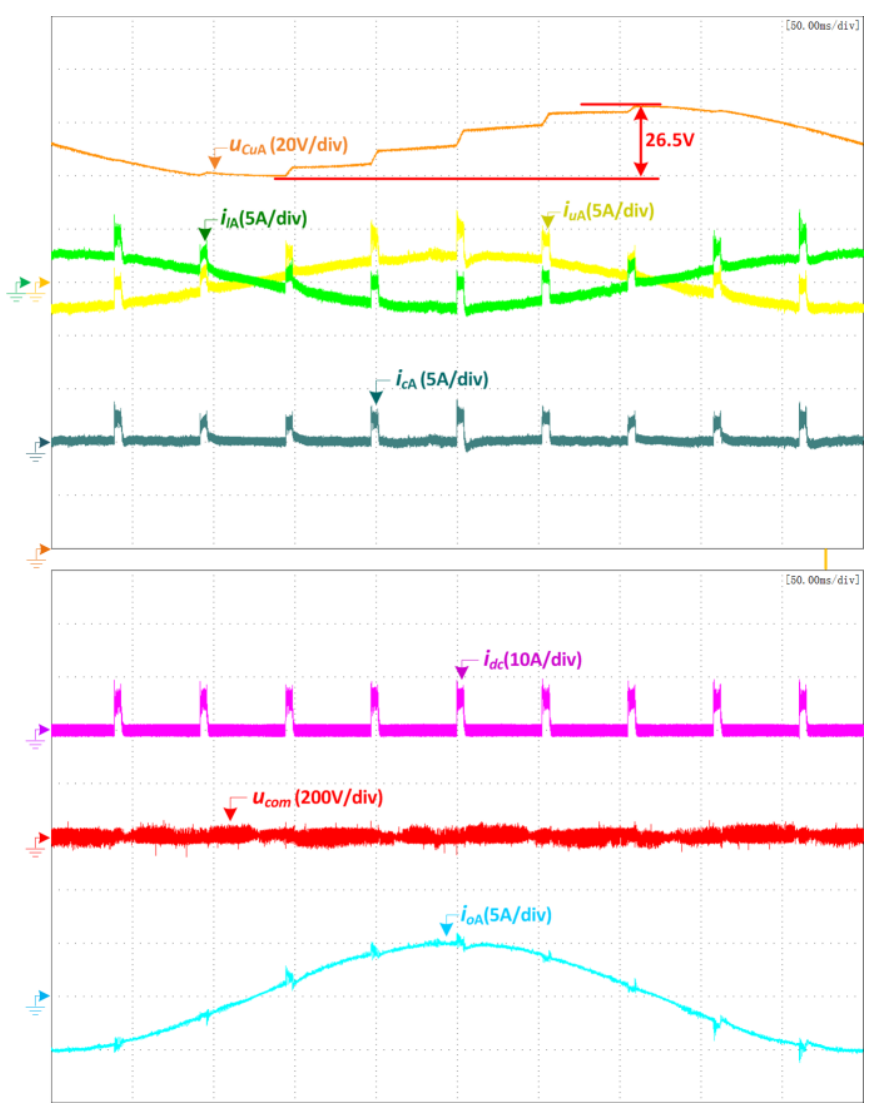

Fig. 18. Experimental waveforms of hybrid MMC at $2 \mathrm{~Hz}$.

From Figs. 17 and 18, we can also observe that the arm current amplitudes were around 5A and the common mode voltage was small, within $\pm 100 \mathrm{~V}$. Neither overcurrent nor overvoltage problems existed.

\section{CONCLUSION}

To overcome the key technical issue of using modular multilevel converters (MMC) in variable-speed drives, this paper proposed a new hybrid MMC topology which can significantly reduce the capacitor voltage ripple, particularly at low motor speeds, while providing rated torque to the $\mathrm{AC}$ machines. Other merits of this topology are that it does not cause any current or voltage overrating of the converter; it does not introduce higher conduction and switching losses; it can offer a degree of dc link short circuit protection; and it does not bring about common-mode voltage nor bearing current which would harm the motor. The operating principle and control schemes for this hybrid MMC were discussed. The validity and effectiveness were confirmed by simulation and experimental results. This topology will be valuable for promoting the use of MMC in medium-voltage motor drives.

\section{REFERENCES}

[1] S. Kouro, M. Malinowski, K. Gopakumar, J. Pou, L. G. Franquelo, B. $\mathrm{Wu}$, J. Rodriguez, M. A. Perez, and J. I. Leon, "Recent advances and industrial applications of multilevel converters," IEEE Trans. Ind. Electron., vol. 57, no. 8, pp. 2553-2580, Aug. 2010.

[2] J. Rodriguez, S. Bernet, P. K. Steimer, and I. E. Lizama, "A survey on neutral-point-clamped inverters," IEEE Trans. Ind. Electron., vol. 57, no. 7, pp. 2219-2230, Jul. 2010.

[3] T. A. Meynard, H. Foch, P. Thomas, J. Courault, R. Jakob, and M. Nahrstaedt, "Multicell converters: basic concepts and industry applications," IEEE Trans. Ind. Electron., vol. 49, no. 5, pp. 955-964, Oct. 2002.

[4] P. W. Hammond, "A new approach to enhance power quality for medium voltage ac drives," IEEE Trans. Ind. Appl., vol. 33, no. 1, pp. 202-208, Jan./Feb. 1997.

[5] A. Lesnicar and R. Marquardt, "An innovative modular multilevel converter topology suitable for a wide power range," in Proc. IEEE Power Tech. Conf., Bologna, Italy, Jun. 23-26, 2003, vol. 3.

[6] M. A. Perez, S. Bernet, J. Rodriguez, S. Kouro, and R. Lizana, "Circuit topologies, modeling, control schemes, and applications of modular multilevel converters," IEEE Trans. Power Electron., vol. 30, no. 1, pp. 4-14, Jan. 2015.

[7] S. Debnath, J. Qin, B. Bahrani, M. Saeedifard, and P. Barbosa, "Operation, control, and applications of the modular multilevel converter: a review," IEEE Trans. Power Electron., vol. 30, no. 1, pp. 37-53, Jan. 2015.

[8] A. Korn, M. Winkelnkemper, and P. Steimer, "Low output frequency operation of the modular multi-level converter", in Proc. IEEE ECCE, 2010, pp. 3993-3997.

[9] M. Hagiwara, K. Nishimura, and H. Akagi, "A medium-voltage motor drive with a modular multilevel PWM inverter," IEEE Trans. Power Electron., vol. 25, no. 7, pp. 1786-1799, Jul. 2010.

[10] J. Kolb, F. Kammerer, M. Gommeringer, and M. Braun, "Cascaded control system of the modular multilevel converter for feeding variable-speed drives," IEEE Trans. Power Electron., vol. 30, no. 1, pp. 349-357, Jan. 2015.

[11] M. Spichartz, V. Staudt, A. Steimel, "Modular multilevel converter for propulsion system of electric ships", in IEEE Electric Ship Technologies Symposium (ESTS), 2013, pp. 237-242.

[12] A. Antonopoulos, L. Angquist, S. Norrga, K. Ilves, L. Harnefors, H.-P. Nee, "Modular multilevel converter ac motor drives with constant torque from zero to nominal speed," IEEE Trans. Ind. Appl., vol. 50, no. 3, pp. 1982-1993, May/Jun. 2014.

[13] J. Jung, H. Lee, and S. Sul, "Control of the modular multilevel converter for variable-speed drives," in Proc. IEEE International Conference on 
Power Electronics, Drives and Energy Systems, 16-19, Bengaluru, India, Dec. 2012, pp. 1-6.

[14] K. Wang, Y. Li, Z. Zheng, and L. Xu, "Voltage balancing and fluctuation-suppression method of floating capacitors in a new modular multilevel converter," IEEE Trans. Ind. Electron., vol. 60, no. 5, pp. 1943-1954, May 2013.

[15] M. Hagiwara, I. Hasegawa, and H. Akagi, "Start-up and low-speed operation of an electric motor driven by a modular multilevel cascade inverter," IEEE Trans. Ind. Appl., vol. 49, no. 4, pp. 1556-1565, Jul./Aug. 2013.

[16] Y. Okazaki, M. Hagiwara, and H. Akagi, "A speed-sensorless start-up method of an induction motor driven by a modular multilevel cascade inverter (MMCI-DSCC)," IEEE Trans. Ind. Appl., vol. 50, no. 4, pp. 2671-2680, Jul./Aug. 2014.

[17] J. Jung, H. Lee, and S.-K. Sul, "Control strategy for improved dynamic performance of variable-speed drives with modular multilevel converter," IEEE J. Emerging Sel. Topics Power Electron., vol. 3, no. 2, pp. 371-380, Jun. 2015.

[18] B. Li, S. Zhou, D. Xu, R. Yang, D. Xu, C. Buccella, and C. Cecati, “An improved circulating current injection method for modular multilevel converters in variable-speed drives" IEEE Trans. Ind. Electron., in early access.

[19] S. Debnath, J. Qin, M. Saeedifard, "Control and stability analysis of modular multilevel converter under low-frequency operation," IEEE Trans. Ind. Electron., vol. 62, no. 9, pp. 5329-5339, Sep. 2015.

[20] D. Busse, J. Erdman, R. J. Kerkman, D. Schlegel, and G. Skibinski, "System electrical parameters and their effects on bearing currents," IEEE Trans. Ind. Appl., vol. 33, no. 2, pp. 577-584, Mar./Apr. 1997.

[21] H. Peng, M. Hagiwara, and H. Akagi, "Modeling and analysis of switching-ripple voltage on the DC link between a diode rectifier and a modular multilevel cascade inverter (MMCI)," IEEE Trans. Power Electron., vol. 28, no. 1, pp. 75-84, Jan. 2013.

[22] Z. Li, P. Wang, Z. Chu, H. Zhu, Y. Luo, and Y. Li, "An inner current suppressing method for modular multilevel converters," IEEE Trans. Power Electron., vol. 28, no. 11, pp. 4873-4879, Nov. 2013.

[23] H. Huang, M. Uder, R. Barthelmess, and J. Dorn, "Application of high power thyristors in HVDC and FACTS systems," in 17th Conference of Electric Power Supply Industry (CEPSI), 2008. pp. 1-8.

[24] P. R. Palmer and A. N. Githiari, "The series connection of IGBT's with active voltage sharing," IEEE Trans. Power Electron., vol. 12, no. 4, pp. 637-644, Jul. 1997.

[25] K. Sasagawa, Y. Abe, and K. Matsuse, "Voltage-balancing method for IGBTs connected in series," IEEE Trans. Ind. Appl., vol. 40, no. 4, pp. 1025-1030, Jul./Aug. 2004.

[26] R. Lizana, M. A. Perez, S. Bernet, J. R. Espinoza, and J. Rodriguez, "Control of arm capacitor voltages in modular multilevel converters," IEEE Trans. Power Electron., vol. 31, no. 2, pp. 1774-1784, Feb. 2016.

[27] B. Li, R. Yang, D. Xu, G. Wang, W. Wang, and D. Xu, "Analysis of the phase-shifted carrier modulation for modular multilevel converters," IEEE Trans. Power Electron., vol. 30, no. 1, pp. 297-310, Jan. 2015.

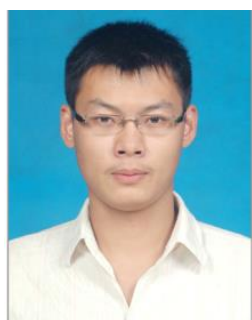

Binbin Li (S'15) was born in 1989. He received the B.S. and M.S. degrees in electrical engineering from the Harbin Institute of Technology, Harbin, China, in 2010 and 2012, respectively, where he is currently working toward the Ph.D. degree.

From July to November 2012, he was with the Silergy Corporation, Hangzhou, China. During 2016, he is a Visiting Researcher at the Department of Electronic and Electrical Engineering, University of Strathclyde, Glasgow, U.K. His research interests include high-power electronics, multilevel converters, control algorithms, and PWM tech

niques.

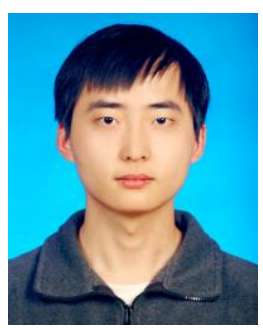

Shaoze Zhou was born in 1993. He received the B.S. in electrical engineering from the Hohai University, Nanjing, China. Since 2015, he has been working toward the Ph.D. degree at Harbin Institute of Technology, Harbin, China.

His research interests include multilevel converters, motor drives, and control algorithms.

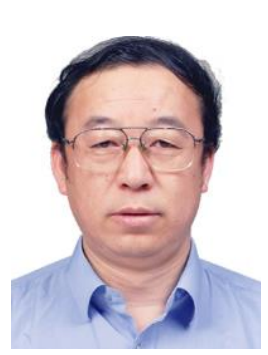

Dianguo Xu (M'97, SM'12) received the B.S. degree in Control Engineering from Harbin Engineering University, Harbin, China, in 1982, and the M.S. and $\mathrm{Ph} . D$. degrees in Electrical Engineering from Harbin Institute of Technology (HIT), Harbin, China, in 1984 and 1989 respectively.

In 1984, he joined the Department of Electrical Engineering, HIT as an Assistant Professor. Since 1994, he has been a Professor in the Department of Electrical Engineering, HIT. He was the Dean of School of Electrical Engineering and Automation, HIT, from 2000 to 2010. He is currently the Vice President of HIT. His research interests include renewable energy generation technology, multi-terminal HVDC system based on VSC, power quality mitigation, speed sensorless vector controlled motor drives, high performance PMSM servo system. He has published over 600 technical papers.

Prof. $\mathrm{Xu}$ is an Associate Editor of the IEEE Transactions on Industrial Electronics and the IEEE Journal of Emerging and Selected Topics in Power Electronics. He also serves as Chairman of IEEE Harbin Section.

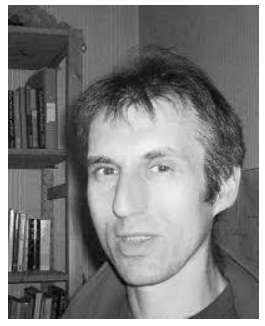

Stephen J. Finney received the M.Eng. degree in electrical and electronic engineering from Loughborough University of Technology, Loughborough U.K., in 1988 and the Ph.D. degree in electrical engineering from Heriot-Watt University, Edinburgh, U.K., in 1994. From 1994 to 2005 he was a member of the academic staff at Heriot-Watt University. In 2005 he moved to the University of Strathclyde, Glasgow, U.K., where he is currently a Professor with the Institute of Energy and Environment, specializing in power electronic systems. His research interests include power electronics for high power applications and the use of power electronics for power transmission and distribution. He has published extensively in IEEE and IEE journals.

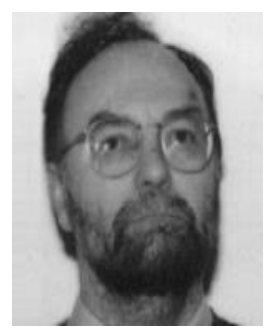

Barry W. Williams received the M.Eng.Sc. degree from the University of Adelaide, Australia, in 1978, and the Ph.D. degree from Cambridge University, Cambridge, U.K., in 1980. After seven years as a Lecturer at Imperial College, University of London, U.K., he was appointed to a Chair of Electrical Engineering at Heriot-Watt University, Edinburgh, U.K, in 1986. He is currently a Professor at Strathclyde University, UK. His teaching covers power electronics (in which he has a free internet text) and drive systems. His research activities include power semiconductor modelling and protection, converter topologies, soft switching techniques, and application of ASICs and microprocessors to industrial electronics. 